\title{
Ecomorphological and phylogenetic controls on sympatry across extant bats
}

\author{
Jeff J. Shi ${ }^{1,2}$ (D) | Erin P. Westeen ${ }^{1,2}$ | Nathan T. Katlein ${ }^{3}$ | Elizabeth R. Dumont ${ }^{4,5}$ | \\ Daniel L. Rabosky ${ }^{1,2}$
}

${ }^{1}$ Museum of Zoology, University of Michigan, Ann Arbor, Michigan

${ }^{2}$ Department of Ecology and Evolutionary Biology, University of Michigan, Ann Arbor, Michigan

${ }^{3}$ Department of Biology, University of South Alabama, Mobile, Alabama

${ }^{4}$ School of Natural Sciences, University of California Merced, Merced, California

${ }^{5}$ Department of Biology, University of Massachusetts Amherst, Amherst,

Massuchusetts

\section{Correspondence}

Jeff J. Shi, Ecology and Evolutionary Biology, University of Michigan, Ann Arbor, MI.

Email: jeffjshi@umich.edu

Editor: Isabel Sanmartín

\begin{abstract}
Aim: Macroecological patterns of sympatry can inform our understanding of how ecological and evolutionary processes govern species distributions. Following speciation, both intrinsic and extrinsic factors may determine how readily sympatry occurs. One possibility is that sympatry most readily occurs with ecological divergence, especially if broad-scale co-occurrence is mediated by niche differentiation. Time since divergence may also predict sympatry if hybridization and gene flow lead to the collapse of species boundaries between closely related taxa. Here, we test for ecological and phylogenetic predictors of sympatry across the global radiation of extant bats.

Location: Global.
\end{abstract}

Taxon: Bats (Order Chiroptera).

Methods: We used a combination of linear mixed-modelling, simulations and maximum-likelihood modelling to test whether phylogenetic and ecomorphological divergence between species predict sympatry. We further assess how these relationships vary based on biogeographic realm.

Results: We find that time since divergence does not predict sympatry in any biogeographic realm. Morphological divergence is negatively related to sympatry in the Neotropics, but shows no relationship with sympatry elsewhere.

Main conclusions: We find that bats in most biogeographic realms co-occur at broad spatial scales regardless of phylogenetic similarity. Neotropical bats, however, appear to co-occur most readily when morphologically similar. To the extent that pairwise phylogenetic and morphological divergence reflect ecological differentiation, our results suggest that abiotic and environmental factors may be more important than species interactions in determining patterns of sympatry across bats.

\section{KEYWORDS}

Chiroptera, ecomorphology, evolutionary ecology, macroecology, macroevolution, sympatry

\section{1 | INTRODUCTION}

Species' geographic distributions and their ranges reflect the interplay between ecological processes and evolutionary patterns (Grossenbacher, Briscoe Runquist, Goldberg, \& Brandvain, 2015; Ricklefs, 2007). In many ways, geographic distributions are unifying units of macroecology and macroevolution, as they are determined by interactions with other species and the environment, and can govern both speciation and extinction. The extent and spatial configuration of species ranges can be controlled by ecological factors, including species interactions (Louthan, Doak, \& Angert, 2015; Sexton, McIntyre, Angert, \& Rice, 2009), abiotic 


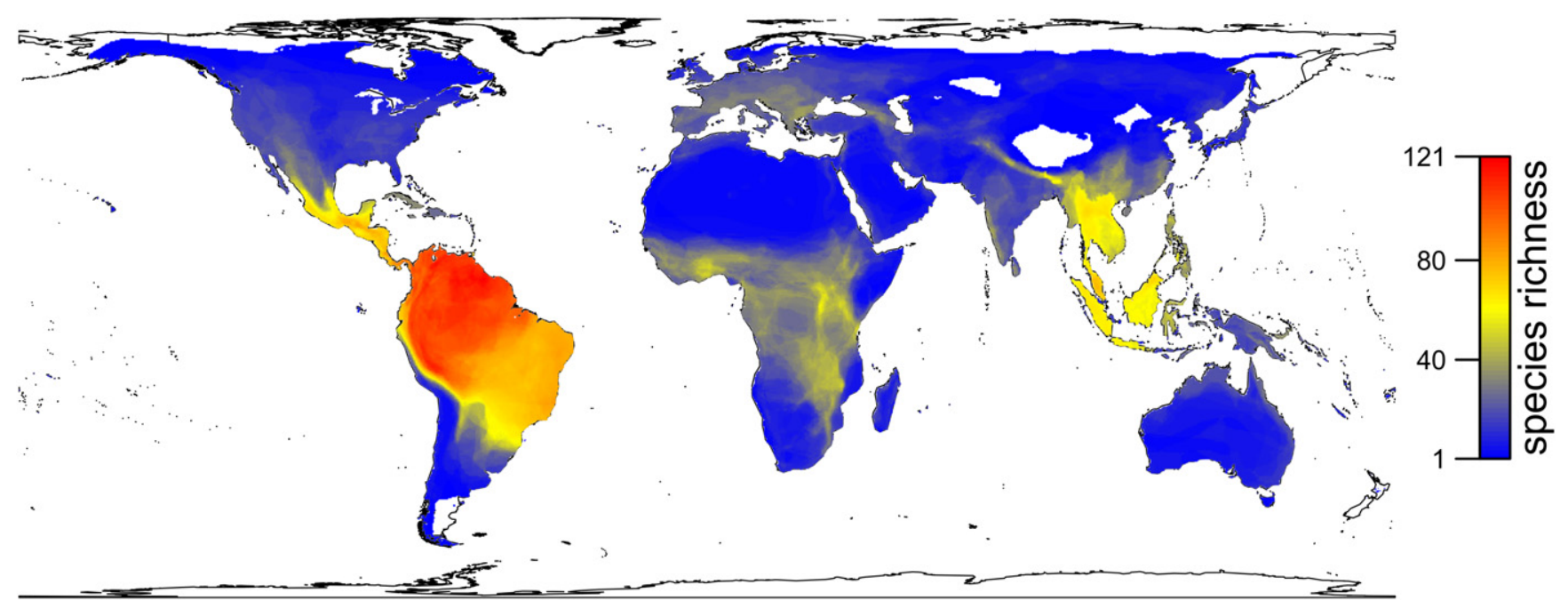

FIGURE 1 Global richness of extant bats, based on 696 range polygons used for this study. Warmer colours represent higher species richness. Regional diversity of bats is highest in the tropics and peaks in the western Amazon basin and eastern slopes of the Andes [Colour figure can be viewed at wileyonlinelibrary.com]

characteristics of the environment (Terribile, Diniz-Filho, Rodríguez, \& Rangel, 2009) and dispersal (Jønsson et al., 2016). Teasing apart these factors is central to macroecological and macroevolutionary research, especially as researchers strive to understand how ecological processes like competition may change distributions and community compositions over macroevolutionary time (Pigot \& Tobias, 2013, 2014).

The configuration of species ranges can reflect patterns of sympatry across species, where "sympatry" refers to broad-scale spatial overlap between species regardless of whether they co-occur in local syntopy. Sympatry at this scale can have multiple controlling factors. The probability of broad-scale sympatry could be dependent on competitive interactions that lead to character displacement and niche divergence (Brown \& Wilson, 1956; Cardillo \& Warren, 2016; Stuart \& Losos, 2013), or even to local extinction due to competitive exclusion (Bengtsson, 1989; Connell, 1972; Silvestro, Antonelli, Salamin, \& Quental, 2015). These general hypotheses invoke stabilizing mechanisms (sensu Chesson, 2000) as a link between divergence and sympatry. Broad-scale sympatry could also be unrelated to resource competition, and instead occurs only in the absence of hybridization, which collapses incipient species (Grant \& Grant, 1997; Taylor et al., 2006). If divergence and reproductive isolation generally increase with time, and if those factors are important controls on sympatry, then we should expect to find a positive correlation between phylogenetic divergence and the probability of sympatry (Barraclough \& Vogler, 2000).

Other models also raise the possibility that greater ecological divergence does not predict extant sympatry. Instead, sympatry can reflect the sorting of regional species pools into communities based on habitat. Species may be more likely to co-occur at low levels of divergence if environmental filtering selects for species with phylogenetically conserved traits (Cavender-Bares, Kozak, Fine, \& Kembel, 2009; Graham \& Fine, 2008; Webb, 2000) and are thus not structured by present-day competitive interactions (McPeek \& Brown, 2000). Some traits may also reflect equalizing mechanisms that reduce fitness differences among organisms (Adler, HilleRisLambers, \& Levine, 2007; Chesson, 2000), and thus would promote sympatry among more similar taxa.

The relationships among sympatry and phylogenetic or phenotypic divergence are unknown across much of the tree of life. Sympatry and divergence are positively related in birds, suggesting a link between local species interactions and broad-scale distributions (Pigot \& Tobias, 2013; Weir \& Price, 2011). Many nonvolant mammalian clades, however, exhibit no relationship between sympatry and phylogenetic divergence (Fitzpatrick \& Turelli, 2006). Such a pattern could indicate that ecological divergence accumulates rapidly in these groups, or that in many cases, sympatric species are not syntopic and do not interact ecologically.

Extant bats (Order Chiroptera) are particularly tractable for exploring the influences of species interactions, phylogeny, and patterns of sympatry at a macroecological scale because of their cosmopolitan distribution and the breadth of their diversity (Jones, Bininda-Emonds, \& Gittleman, 2005; Shi \& Rabosky, 2015; Simmons, 2005). Their potential for high dispersal via flight may mean that species interactions are more important than landscape or edaphic features for predicting spatial patterns. As regional dispersal can also erode any local signals of species interactions, bats may be a system where sorting patterns play a disproportionate role.

Bats feed on a wide variety of resources, including arthropods, vertebrates, fruits and nectar (Nowak, 1994; Simmons \& Conway, 2003). Competition for these resources structures many bat communities at local scales, such as within Neotropical savannas (Aguirre, Herrel, van Damme, \& Matthysen, 2002; Estrada-Villegas, McGill, \& Kalko, 2012). There is also evidence that some bat communities are structured by echolocation frequency and trophic ecology (Findley \& 
Black, 1983; Moreno, Arita, \& Solis, 2006; Siemers \& Schnitzler, 2004). However, we do not know the extent to which competitive interactions for resources among bats are important controls on sympatry, or how these controls may vary across global bat diversity (Figure 1).

Bat ecology is tightly coupled with morphology; this is especially well-studied with trophic ecology and skull morphology. The shape and size of bat skulls reflect the link between physiological performance and the ability to capture and process foods with highly variable mechanical properties (Dumont, 2004; Nogueira, Peracchi, \& Monteiro, 2009; Santana \& Cheung, 2016; Santana, Dumont, \& Davis, 2010; Saunders \& Barclay, 1992), and thus are often used as proxies for ecological metrics in the absence of observational and experimental data. In some families, skull morphology is also closely tied with echolocation ability, another dimension of trophic ecology (Curtis \& Simmons, 2017; Santana \& Lofgren, 2013). While relative performance data among coexisting bat species are rare, morphological divergence is often considered to be at least one predictor of ecological divergence.

In this study, we test whether overall, broad patterns of sympatry can be predicted by phylogenetic and/or morphological divergence across extant bats. With range data and museum specimens, we use phylogenetic linear mixed-modelling to test predictors of sympatry, and a maximum-likelihood framework to model the probability of sympatry as a function of age and morphological distance. We explore the influence of phylogenetic dependence on our range data, and propose a general framework for testing if sympatry can be related to various metrics of divergence.

\section{MATERIALS AND METHODS}

\section{1 | Overall framework and scope}

We explored how sympatry varies with two pairwise metrics of divergence: time to the most recent common ancestor, and ecomorphological divergence as represented by Euclidean distances between skulls in morphospace. We focused on the binary presence $(0 / 1)$ of broad-scale sympatry, given a threshold of continuous range overlap (a percentage) in a species pair. Our framework involved three approaches: (1) pairwise linear mixed-models to test divergence predictors of sympatry, accounting for random effects of phylogeny and species identity; (2) maximum-likelihood modelling of how multiple parameters of sympatry may vary with pairwise divergence among sister taxa (sensu Pigot \& Tobias, 2013); (3) randomizations that infer the null distributions of sympatry across species pairs given no relationship with divergence. For the pairwise linear mixed-models (approach 1), we integrated data from all species pairs. In the maximum-likelihood models (approach 2), we focused on a subset of sister species, where we might expect species interactions to be strongest. All analyses used the species-level Chiroptera phylogeny of Shi and Rabosky (2015) (Appendix S2), which contains 812 of the roughly 1,300 extant species of bats.
All analyses were divided into biogeographic realms, representing regional pools of species that could reasonably co-occur in the absence of constraints on sympatry. We used World Wildlife Fund (WWF) realms (Olson et al., 2001), though we combined the small Oceanic and Australasian realms and excluded bats endemic to Madagascar, Seychelles, and Comoros from the Afrotropics. We divided our analyses to infer how predictors of sympatry vary by region to capture species pools that sort into communities (Lessard, Belmaker, Myers, Chase, \& Rahbek, 2012), and to minimize one potential source of biogeographic bias. To illustrate this, consider the different species pools between the Indian Ocean islands and the mainland Afrotropics. Even if taxa in these two regions are rarely found in sympatry due to ancient vicariance, pairwise allopatry states would be repeatedly counted in all comparisons between descendant species of the two regions, regardless of the time since divergence. This would artificially bias relationships between divergence and sympatry in a negative direction (e.g. greater divergence being correlated with lower probabilities of sympatry; see Appendix S3).

\section{2 | Morphological data}

We took nine linear measurements (Appendix S4: Table S3) from bat skulls at the University of Michigan Museum of Zoology (UMMZ) and the American Museum of Natural History (AMNH). These measurements followed Dumont (2004) and Dumont et al. (2012), who linked ecomorphology and diversification in the family Pteropodidae and the superfamily Noctilionoidea. From species-level averaged measurements, we calculated pairwise Euclidean distances in ninedimensional trait space between all pairs as our metric of pairwise ecomorphological divergence.

We targeted 241 species across 14 of the 20 extant families of bats based on available specimens, representing roughly $30 \%$ of the phylogeny.

\section{3 | Spatial data and sympatry}

We used species ranges from the IUCN's Red List of Threatened Species (IUCN, 2016), though with modifications to the superfamily Noctilionoidea (Appendix S5). We targeted available range polygons based on our phylogeny.

With these polygons, we used the rgeos and maptools $\mathrm{R}$ packages to code sympatry state for all pairs of extant bat species in the spatial dataset. We first calculated geographic range overlap with the Szymkiewicz-Simpson coefficient, or the sum area of overlap divided by the range size of the species with the smaller range, for each species pair. We then designated each pair of bat species as sympatric or allopatric based on a threshold of $20 \%$ range overlap (as in Pigot \& Tobias, 2013), though we also report results from more conservative thresholds (Appendix S6). We decomposed our data into binary states, as opposed to continuous overlap, as the latter metric is more sensitive to assumptions of speciation mode (Phillimore et al., 2008). 


\subsection{Phylogenetic linear mixed-modelling}

To test if overall pairwise sympatry within biogeographic realms is predicted by divergence, we used phylogenetic linear mixed-models (PLMMs). PLMMs are particularly flexible for their ease of interpretation and implementation in a standard mixed-modelling framework, and the ability to test for distinct fixed and random predictor(s) on response variable(s). Furthermore, they can easily incorporate paired, continuous and categorical data.

We used Markov chain Monte Carlo to simulate posterior distributions of model parameters using the MCMCgImm R package (Hadfield, 2010). Our PLMMs took the general form:

$S_{i, j}=\beta X_{i, j}+Z_{1} u_{i, j}+Z_{2, i}+Z_{2, j}$. Our response variable $S$ corresponded to the probability of sympatry for a given species pair $i$ and $j$ and was related to the observed data (sympatry/allopatry) using a probit ("threshold") link function. We tested for a vector of fixed effects $\beta$, given a matrix $X$ of divergence metric(s) between species $i$ and $j$. We then incorporated two distinct classes of random effects $Z$ into our PLMMs: the hierarchical effect of phylogenetic structure $\left(Z_{1}\right)$ and species identity $\left(Z_{2}\right)$ (Hadfield \& Nakagawa, 2010; Tobias et al., 2014). $Z_{1}$ accounted for the possibility that fixed effects depend on phylogenetic node structure $\left(u_{i, j}\right)$ and thus subclade identity, while $Z_{2}$ accounted for the multiple times each unique species $i$ and $j$ was represented in our datasets. We ran all models with a standard inverse-gamma prior on the variance structure of our random effects (Hadfield, 2010). We checked all MCMC output for autocorrelation at different levels of sample thinning, while also confirming high (variance $>1,000$ ) effective sample sizes.

As we did not have representative morphological data for every species, we ran two groups of PLMMs with varying $\beta$ and $X$ vectors. The first set of PLMMs only tested for $\beta_{1}$, the effect of phylogenetic divergence (in mya) on pairwise sympatry, with separate models for each realm. For each model, we simulated the posterior distributions of model parameters using 20 million generations of MCMC simulation, sampling every 10,000 generations, with $10 \%$ discarded as a burn-in.

The second set of PLMMs tested for three fixed effects: (1) $\beta_{1}$, (2) $\beta_{2}$ : the effect of ecomorphological divergence and (3) $\beta_{3}$ : the interaction of both divergence metrics. $\beta_{3}$ accounted for the possibility that the strength of ecomorphological control depends on time since divergence. Given the limited sampling of our morphological data, this second set was divided into just the Nearctic and Neotropical realms, as well as the combined New World. We simulated this second set of posterior distributions of model parameters using 10 million generations of MCMC simulation, sampled every 5,000 generations, with $10 \%$ discarded as burn-in, as these were much smaller datasets.

\section{5 | Modelling the probability of sympatry}

We further used a maximum-likelihood (ML) framework to compare models where multiple parameters that govern the relationship between sympatry and divergence can be estimated. We fit models in which the probability of sympatry explicitly varies with

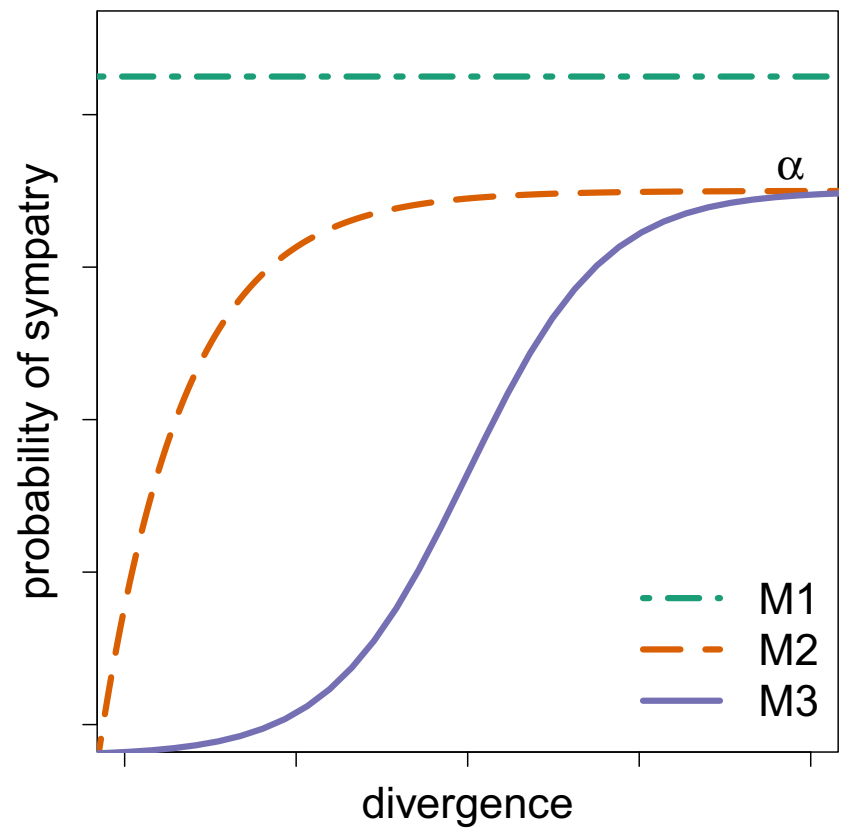

FIGURE 2 Three models for how the probability of sympatry $(\theta)$ can vary as a function of either phylogenetic or morphological divergence. From top to bottom: M1, a model where $\theta$ is independent of the evolutionary or morphological divergence between taxa; M2, where $\theta$ approaches a limiting value $\alpha$; 3 , where $\theta$ logistically varies with divergence and also asymptotically approaches a limiting value $\alpha$ [Colour figure can be viewed at wileyonlinelibrary.com]

phylogenetic ( $t$, time in mya) and/or morphological ( $d$, pairwise Euclidean distance) divergence (Figure 2). We tested covariates independently, and also in interaction $(t d)$, to account for scenarios where morphological divergence has the most dramatic effect in close relatives. We restricted these analyses to sister taxa represented in the tree, as we may expect to find the strongest signal of divergence among young pairs. Although these pairs may not be true sisters, this restriction accounted for phylogenetic non-independence of data; this general approach was analogous to that of Pigot and Tobias (2013). We performed the following analyses for all measured sister species pairs, and for the subset composed of New World pairs, where the bulk of our morphological data are represented.

For these analyses, we treated the probability of sympatry as a binomially distributed random variable with a single parameter $\theta$. The likelihood $L$ of observing any combination of allopatry (0) and sympatry (1) states across pairs of species $i$ and $j$, in a set of $n$ species $Y$, was thus denoted by $L=\prod_{i, j=1}^{n} \operatorname{Pr}\left(Y_{i, j} \mid \theta\right)$, where $\operatorname{Pr}\left(Y_{i, j} \mid \theta\right) \sim \operatorname{binom}(\theta)$. $\theta$, in turn, was governed by three potential models of sympatry (Figure 2). For M1, $\theta$ was treated as a constant. This model served as our null hypothesis: under this model, the ML estimate for the probability of sympatry is simply the percentage of sympatric pairs in a given set $Y$.

In $\mathrm{M} 2, \theta$ varied as an exponential decay function with $t, d$ or $t d$ as follows (written for $t$ alone): $\theta=\alpha\left(1-e^{-k t}\right)$. M2 reflected scenarios in which pairwise sympatry varied with divergence. Because $\theta$ 


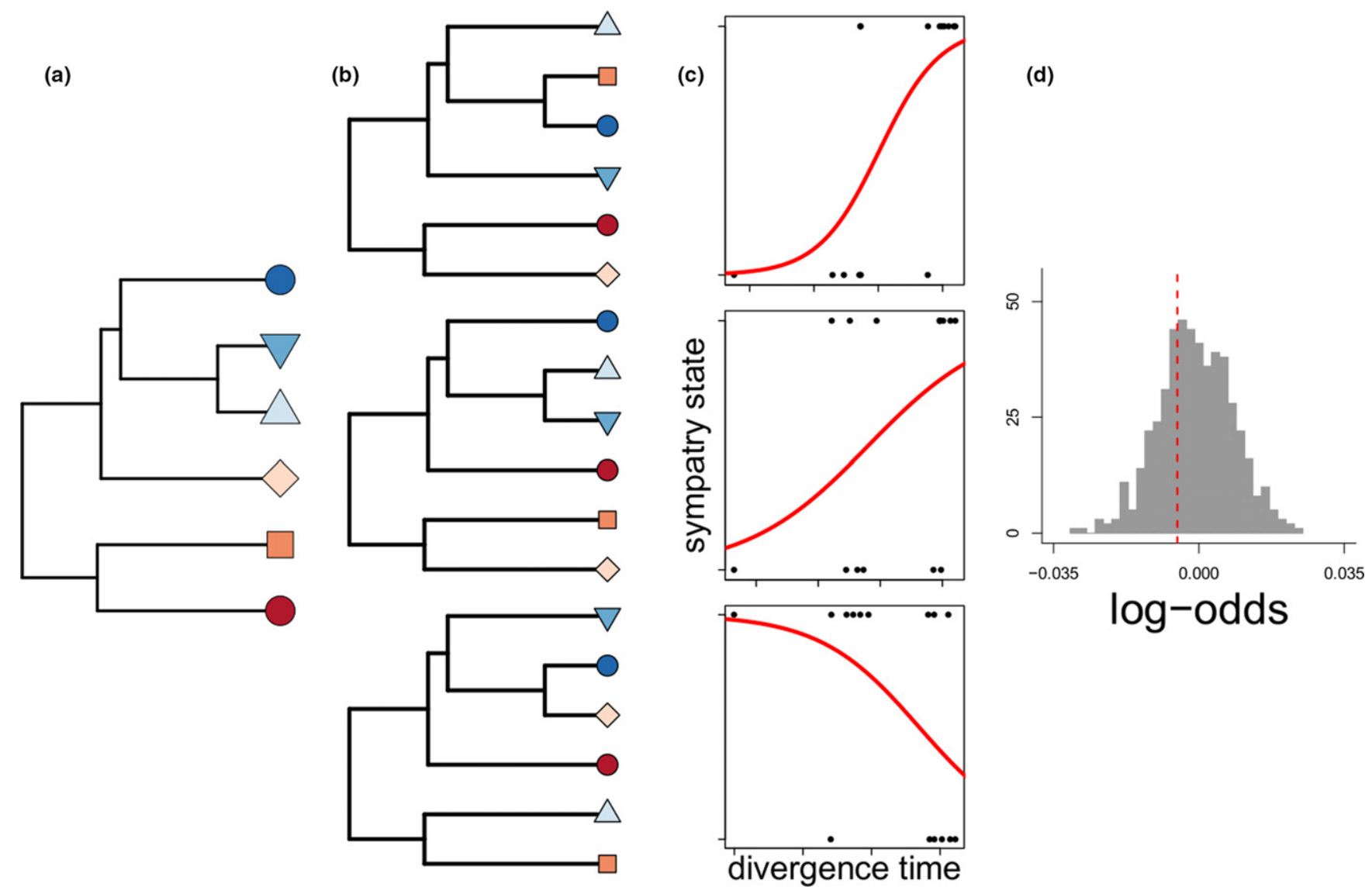

FIGURE 3 A schematic of our range randomization approach used to test the relationship between sympatry and the time since divergence. For each realm, we took the (a) phylogeny of all bat species endemic to that realm and (b) randomized species and range identity while holding the tree constant. For each of these randomizations, we calculated (c) the logistic regression and associated log-odds between divergence time and sympatry state. Repeating (b) and (c) 500 times created (d) a null distribution of relationships, shown here as the logarithm of the odds ratio between divergence time and sympatry state. This null distribution was then compared to the empirical value for that realm, indicated by the dashed line [Colour figure can be viewed at wileyonlinelibrary.com]

approaches an unfixed asymptote $\alpha$, which is a parameter estimated from the data, the model also accounted for the biological reality that some species pairs will simply never become sympatric due to geographic or historical constraints (Figure 2). The rate parameter $k$, which reflects how rapidly $\theta$ approaches $\alpha$, was also estimated from the data, where $\mathrm{M} 2$ reduces to $\mathrm{M} 1$ as $k$ approaches infinity.

In our final model (M3), $\theta$ varied logistically with $t, d$ or $t d$ as follows (written for $t$ alone): $\theta=\frac{\alpha}{1+e^{-k(t-w)}}$. M3 represented a scenario analogous to one proposed by Pigot and Tobias (2013, 2014), where $\theta$ is correlated with time and/or ecomorphology, but includes a lag or delay parameter ( $w$ ) before sympatry is readily attained (Figure 2). This $w$ parameter may represent a minimum threshold of morphological divergence to avoid competition, or a minimum age threshold to avoid hybridization, among other possibilities. In this case, $\alpha, w$, and the rate parameter $k$ were all estimated from the data, where $M 3$ will also reduce to $M 1$ when $w=0$ and $k$ approaches infinity.

We fitted all seven potential models to sister species data using the bbmle $\mathrm{R}$ package. We tested overall model fit using the corrected Akaike information criterion (AICc). Our model setup also allowed us to explicitly test hypotheses using likelihood-ratio tests within the three groups of related models (one group for each metric of divergence $t, d$ or $t d$, where M1 was always the null hypothesis of no relationship between divergence and $\theta$ ). Given our sample sizes of sister species pairs, we also explored false-positive rates using randomly simulated datasets (Appendix S7).

\subsection{PLMM and ML model validation}

We applied both our PLMM and ML model-fitting approaches to the phylogenetic, morphological and spatial data of sister species pairs of Neotropical ovenbirds (Family Furnariidae) from Pigot and Tobias (2013), who concluded that ecomorphological and phylogenetic divergence affected the rate at which species pairs became sympatric (Appendix S9). By using the same data as Pigot and Tobias (2013), we tested whether our analytical framework could recover similar relationships between divergence and sympatry as reported in their study.

\section{7 | Sympatry-age relationships}

Finally, we inferred a null distribution of the relationship between pairwise sympatry state and time since divergence by using a set of 
randomizations (Figure 3). We randomly assigned species (and thus ages) to ranges, for each extant bat, and then fit a logistic model for sympatry as a function of age. This randomization process, representing a model where the pattern of sympatry across bats is random with respect to divergence time, was repeated 500 times. These randomizations established a distribution of randomized logodds from logistic models, and we compared this to the empirical age-overlap relationship. We performed these randomization tests for each of the six WWF biogeographic realms.

\section{RESULTS}

\section{1 | Data summary}

Overall, we report results for 696 bats with spatial data that are included in our phylogenetic tree. We measured 1,073 adult specimens at the UMMZ and combined these data with the previously published AMNH data of Dumont et al. (2012) (mean specimens/ species $=3.86, S D=3.53$ ).

Regional pairwise sympatry among bats is consistently high, given a $20 \%$ threshold of overlap (Table S1; weighted average: $42.2 \%$ of pairs are sympatric). This does not appear to be correlated with regional species diversity or realm size, as even the relatively low diversity but large Nearctic realm has over $50 \%$ of its species pairs in sympatry. In both New World realms (the Neotropics and the Nearctic), 50\% or more of species pairs are sympatric, with average overlap percentages near $40 \%$. We note that in all realms but the Palaearctic, average overlap is above our base threshold for sympatry.

\subsection{PLMM results}

MCMCgImm returns $\mathrm{pMCMC}$ values, which are two-tailed calculations of the proportion of simulations where fixed effects differ from zero. We use these to assess the significance of fixed effects in PLMMs, and find that time since divergence does not significantly predict sympatry in any realm (Table 1). We can also use highest posterior density intervals and credibility intervals to evaluate our posterior distribution, but in our analyses, all these methods are concordant (see Supporting Information).

TABLE 1 PLMM results for the effect of age $\left(\beta_{1}\right)$ alone on pairwise patterns of sympatry (at a $20 \%$ overlap threshold) for all pairs of bat species with spatial data, divided into WWF biogeographic realms. Posterior means and pMCMC values (see Results) are included

\begin{tabular}{lll} 
Realm ( $N$ ) & $\boldsymbol{\beta}_{1}$ posterior mean & $\boldsymbol{\beta}_{1}$ pMCMC \\
\hline Afrotropics (78 species) & -0.016 & 0.060 \\
\hline Indomalaya (175 species) & -0.008 & 0.083 \\
\hline Nearctic (40 species) & -0.018 & 0.182 \\
\hline Neotropics (235 species) & -0.012 & 0.336 \\
\hline Oceania \& Australasia (82 species) & -0.008 & 0.481 \\
\hline Palaearctic (70 species) & -0.009 & 0.209 \\
\hline
\end{tabular}

In the New World bats, when we incorporate ecomorphological divergence, we find that there are notable differences between Nearctic and Neotropical bats. There are no significant effects of divergence in the Nearctic. However, we recover significant evidence for a negative relationship between ecomorphological divergence and binary sympatry state in the Neotropics (Table 2; Figure 4). While there is some uncertainty in the specific relationship - particularly in a threshold of ecomorphological divergence that makes sympatry less likelythere is extremely strong support for a negative signal in the data (Figure 4b). This negative relationship does not appear to be driven by divergent outliers, as we recover concordant results with an analysis on a smaller subset of our data (Figure 4c, Appendix S15). Across the entire New World (Nearctic + Neotropics), the interaction of phylogeny with ecomorphology has a negative effect on sympatry, though the two variables are not significant predictors independently (Table 2). These negative relationships imply that sympatry is actually less likely as divergence increases.

If we subsample by varying the threshold overlap percentage for sympatry, we generally recover concordant results in our PLMMs, implying that our main analyses are conservative in estimating predictors of sympatry (Appendix S6).

\subsection{ML models of the probability of sympatry}

We fit our ML models of sympatry to 67 sister species pairs, as well as $53 \mathrm{New}$ World sister species pairs. A simple, null model where all species pairs share a common probability of sympatry, regardless of any type of divergence, was the best-fitting model (Appendix S7).

\section{4 $\quad$ PLMM and ML model validation}

We recover, as do Pigot and Tobias (2013), positive effects of both divergence time and ecomorphology on sympatry in furnariid sister species with both PLMMs and our ML models (Appendix S9). We specifically find strong evidence for models with a lag time, further suggesting that species interactions mediate sympatry.

\section{5 | Sympatry-age relationships}

In each WWF biogeographic realm, the null distributions of agesympatry relationships (calculated from range randomizations as logodds from logistic regressions between sympatry state and time since divergence, as described in Figure 3) are centred around 0, as expected. The empirical age-sympatry relationship does not appear to significantly deviate from the null distribution in any realm, though it skews slightly negative in the Afrotropics (Appendix S11).

\section{DISCUSSION}

\subsection{Divergence time and sympatry}

We find no significant effects of age on pairwise patterns of sympatry (Table 1). We also find that there is no significant difference 
TABLE 2 PLMM results for the effects of age $\left(\beta_{1}\right)$, ecomorphological divergence $\left(\beta_{2}\right)$, and combined age and ecomorphological divergence $\left(\beta_{3}\right)$ on pairwise patterns of sympatry (at a $20 \%$ overlap threshold) for all pairs of bat species with both types of divergence data. These pairs are divided according to realm. Posterior means and $\mathrm{pMCMC}$ values are included, and bolded when pMCMC $<0.05$. Note that some species are part of the species pools of both realms

\begin{tabular}{lllllll} 
Realm $(\boldsymbol{N})$ & $\boldsymbol{\beta}_{\mathbf{1}}$ posterior mean & $\boldsymbol{\beta}_{\mathbf{1}} \mathrm{pMCMC}$ & $\boldsymbol{\beta}_{\mathbf{2}}$ posterior mean & $\boldsymbol{\beta}_{\mathbf{2}} \mathrm{pMCMC}$ & $\boldsymbol{\beta}_{\mathbf{3}}$ posterior mean & $\boldsymbol{\beta}_{\mathbf{3}} \mathrm{pMCMC}$ \\
\hline Nearctic (34 species) & -0.029 & 0.380 & -0.015 & 0.958 & -0.002 & 0.800 \\
Neotropics (135 species) & -0.018 & 0.203 & -0.091 & $\mathbf{0 . 0 0 9 *}$ & $<0.001$ & 0.621 \\
\hline New World (161 species) & -0.026 & 0.330 & -0.029 & 0.360 & -0.002 & $0.004^{*}$ \\
\hline
\end{tabular}

between a process-neutral null model and any ML model where the probability of sympatry varies with age (Appendix S7). Age is often intrinsic to any explanation for patterns of sympatry, especially given correlations of divergence with time. However, our finding is consistent across all biogeographic realms. Therefore, even though one explanation for this null pattern is that divergence and time are simply not well-correlated in bats, it is unlikely this is true across all families and realms (Appendix S16).

\section{2 | Ecomorphology and sympatry in the New World}

We find no evidence for ecomorphological controls on sympatry among the measured Nearctic bat species, but find that there is a negative relationship between ecomorphological divergence and sympatry among Neotropical bats (Figure $4 b, c)$. We also find a negative interaction effect of age and ecomorphology on sympatry across New World bats as a whole in our PLMMs (Table 2). As noctilionoids are characterized by strong relationships between ecology and highly specialized morphology (Dumont et al., 2012), we may have expected to see the strongest link between divergence and sympatry in this realm. Nevertheless, Neotropical species pairs are more likely co-occur when they are morphologically similar. Multiple hypotheses could explain this pattern, including community assembly via environmental filtering, or within-realm sorting that biases where similar species are most likely to be found (Cavender-Bares et al., 2009; Graham \& Fine, 2008; Leibold \& McPeek, 2006; Webb, 2000). Within noctilionoids, there are numerous examples both of clades that are filtered by resource availability, leading to sympatry among the most similar pairs, and those that assemble into communities based on stabilizing mechanisms (Villalobos \& Arita, 2010). As our morphological data are partial proxies for ecological divergence, a deeper dataset that addresses feeding mechanics and performance may yield a fine-grained picture of how functional divergence relates to co-occurrence within communities.

Despite the significant negative effect of ecomorphology in our PLMMs, our best-fitting ML model is a simple one in which all pairs share a common probability of sympatry regardless of phylogenetic or morphological divergence (Appendix S7). This discrepancy likely reflects a fundamental difference between the two datasets. It is possible that the shorter time-scales associated with sister taxa are insufficient for accumulating enough ecomorphological divergence to influence the processes governing sympatry. Our sister species dataset is also relatively small, and it thus possible that statistical power was lower for these analyses.

The significant New World interaction effect of divergence metrics on sympatry in our PLMMs (Table 2) likely reflects scale and differences between Nearctic and Neotropical bats. Nearctic bats are predominantly insectivorous vespertilionoids, while the Neotropics are dominated by their high richness of noctilionoid bats, which span the full breadth of bat feeding diversity (Nowak, 1994; Simmons, 2005). We can interpret this significant effect as evidence that, at the scale of the entire New World, we are most likely to find morphologically similar and closely related bats in sympatry. This is likely compounded by the fact that morphological divergence among many Neotropical species can be relatively large, and is recent compared with the relatively ancient ( 50 mya) divergence of noctilionoids from Nearctic vespertilionoids (Shi \& Rabosky, 2015).

\section{3 | Sympatry-divergence relationships across extant bats and potential causes}

Multiple interactions beyond resource competition can drive patterns of sympatry. Mutualistic interactions with plants, or predation and parasitism (Mcintire \& Fajardo, 2014; Spiesman \& Inouye, 2014) can govern spatial patterns. Some bat communities, their distributions, and abundances are non-randomly structured with respect to other phenotypic traits, including flight ability and echolocation (Corcoran \& Conner, 2014; Norberg \& Rayner, 1987; Santana \& Lofgren, 2013; Schoeman \& Jacobs, 2003; Siemers \& Schnitzler, 2004), as well as available foraging and roosting habitats (Schoeman \& Jacobs, 2011; Voss, Fleck, Strauss, Velazco, \& Simmons, 2016). These multiple pressures existing in conjunction could mask relationships between skull morphology and sympatry. The framework we develop here is flexible to the integration of other metrics of divergence, including measures of ecological performance that more directly test for competition.

Low competition for resources among bats may also decouple divergence from sympatry, especially if resources like aerial insects are ubiquitous and plentiful at night (Fenton \& Thomas, 1980; Fleming, 1986). Studies that test for resource competition among bats are uncommon, and there is mixed evidence depending on guild, body size and seasonality (Heithaus, Fleming, \& Opler, 1975; Kingston, Jones, Zubaid, \& Kunz, 2000; Swift \& Racey, 1983). Divergence may also occur in situations when species historically co-occurred, but exist presently in allopatry, thereby masking the signature of the sympatry-divergence relationship (Anacker \& Strauss, 2014). 

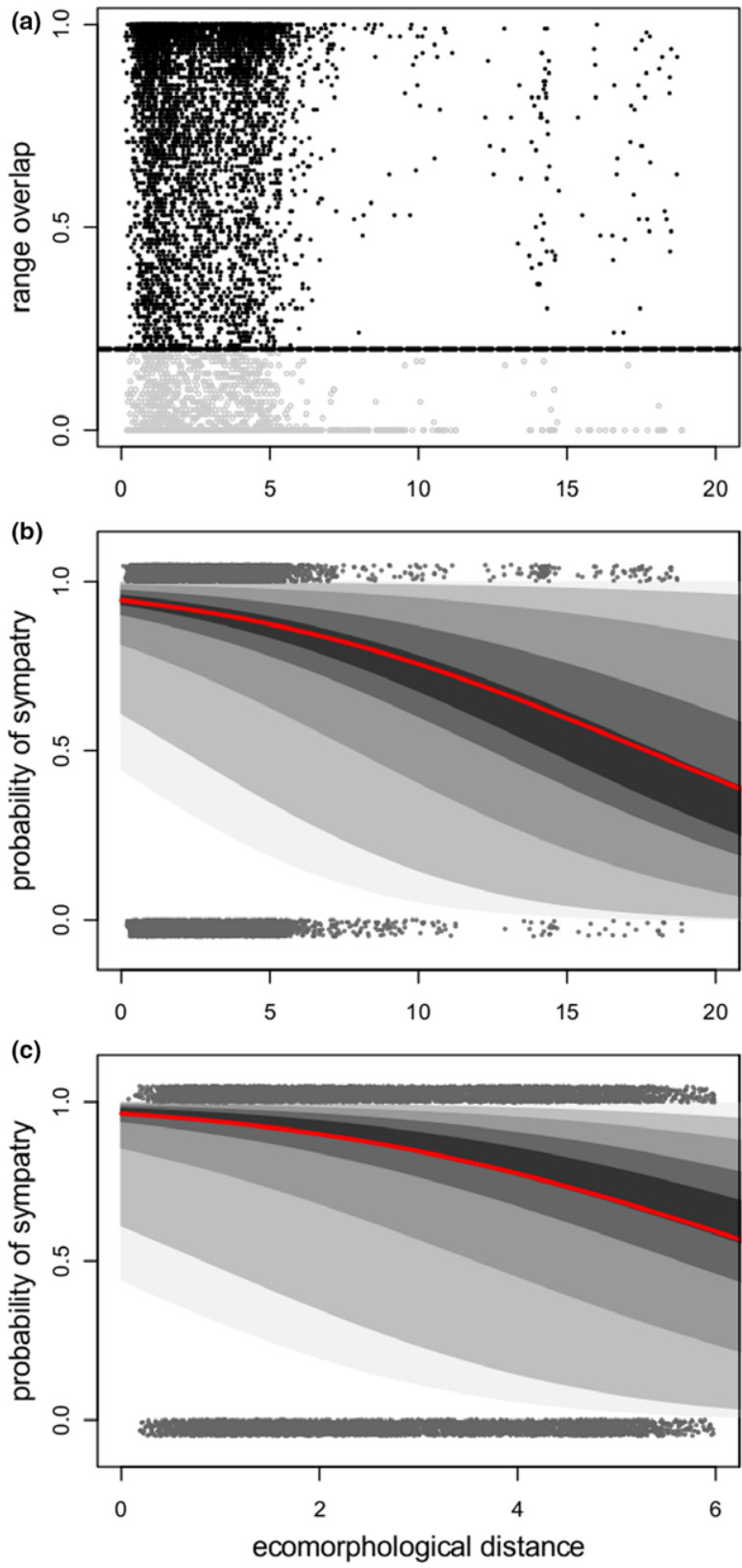

FIGURE 4 (a) Pairwise Euclidean distances (ecomorphology) versus percentage range overlap for all pairs of Neotropical bat species considered in this study ( $N=8,967$ pairs). Pairs above the dotted threshold are considered sympatric for the main analyses of this study. (b) Points denote the same dataset, decomposed into binary sympatry or allopatry states. The curve is the posterior mean PLMM estimate of the relationship between pairwise ecomorphological distance and the probability of sympatry. Progressively darker polygons highlight the $90 \%, 75 \%, 50 \%, 25 \%$ and $10 \%$ credibility intervals around the mean. There is a significant negative relationship between the probability of sympatry and ecomorphological distance, although credible intervals are wide. (c) The same relationship as (b), but fitting the model only to species pairs with morphological distances less than 6.0, which accounts for $95.8 \%$ of all species pairs. This analysis was performed to ensure that the overall negative relationship was not driven by the small number of pairs with very high ecomorphological distance values [Colour figure can be viewed at wileyonlinelibrary.com]
Furthermore, we must also acknowledge that ranges themselves are inherited and non-independent properties of species. While we partially account for this in the random effects of our PLMMs, there is considerable room for the integration of models that simulate range heritability and evolution.

Divergence may also be unrelated to sympatry if abiotic filtering is the dominant process shaping species assemblages at the spatial scales considered here. For example, elevation and water availability (Henry, Barrière, Gautier-Hion, \& Colyn, 2004; McCain, 2007a,b) control syntopy at local scales, but this fine-grained spatial structuring might not translate to regional range overlap. Bat diversity in the Afrotropics, for instance, appears to be highest in the wettest and most humid regions (Figure 1); this pattern may underlie co-occurrence in sympatry. Bat distributions can also vary with temporal and seasonal variation in resource use (Adams \& Thibault, 2006; Kronfeld-Schor \& Dayan, 2003). Abiotic, environmental conditions can also mediate ecological interactions, eroding clear relationships between divergence and sympatry (Chesson, 1986; Dunson \& Travis, 1991). If traits actually underlie fitness differences as opposed to niche differences, then equalizing mechanisms may be the most important promoters of coexistence, which can also result in null or negative relationships between divergence and sympatry (Adler et al., 2007; Chesson, 2000). This seems less likely in bats, where morphological differences are linked to major trophic categories, but is a possibility for other taxa characterized by generally low divergence.

It is also possible that there are trade-offs between mechanisms of divergence and habitat filtering that scale with community and range sizes (Kneitel \& Chase, 2004). Local communities can be overdispersed without this pattern manifesting at the regional scale (e.g. Rabosky, Cowan, Talaba, \& Lovette, 2011). Local and regional scales are also not consistent across organisms and biomes, given differences in dispersal ability (Warren, Cardillo, Rosauer, \& Bolnick, 2014). Finally, processes that control the degree of overlap may be distinct from those that preclude co-occurrence altogether. Even given no relationship between divergence and the presence of sympatry, there may still be a relationship between divergence and the degree of overlap in a subset of sympatric pairs, indicating that once requirements for sympatry are met, range overlap is readily increased.

Our results indicating weak or null effects of phylogenetic distance on regional co-occurrence could also be evidence for alternative modes of speciation, including speciation in sympatry. While speciation in allopatry is often assumed to be the most prevalent mode, sympatric speciation could cloud any signals of divergence upon sympatry (Fitzpatrick \& Turelli, 2006), especially if extant ranges largely reflect the geography of speciation. Reproductive sorting by echolocation frequency has been suggested as a driver of sympatric speciation in some clades of bats (Kingston \& Rossiter, 2004). Considering the generally coarse nature of available range data, allopatric pairs may even appear sympatric, as in cases where isolation depends on microhabitat availability like roosts (Voss et al., 2016). Spatial patterns of bat diversity may also be unrelated to 
divergence if larger ranges are simply more likely to overlap when constrained by continental geography, analogous to the mid-domain explanation for the latitudinal diversity gradient (Colwell \& Lees, 2000). This would also be evidence for dispersal ability as a driver of sympatry across bats, though testing would require higher resolution data on range limits. Dispersal could even erode signals of local competitive exclusion, leading to the appearance of widespread sympatry.

One of the biggest limiting factors to macroecological studies is the quality and accuracy of data. Uncertainty in divergence time estimation can impede efforts to infer the effects of age on extant diversity. The presence of cryptic species may make identification of syntopic species difficult. Furthermore, all studies that use spatial data are sensitive to the accuracy of range maps, which have not been systematically reviewed across Chiroptera, to our knowledge. Ultimately, it is unlikely that ecological interactions scale to macroecological patterns and macroevolutionary dynamics equally across the tree of life. The negative relationship between divergence and co-occurrence across bats is potentially evidence that their diversity is unsaturated (Shi \& Rabosky, 2015), and that they are continuing to radiate into a diversity of ecological niches and biomes.

\section{ACKNOWLEDGEMENTS}

The authors acknowledge M.C. Grundler and P.O. Title for their invaluable assistance and advice regarding statistical and spatial analyses, and three anonymous referees for improving the quality of this paper. J.J.S. also thank L.M. Dávalos, J.P. Drury, J. Hadfield, K. Langstrom, A.L. Pigot, D. Rojas, S. Singhal and H.L. Williams for methodological advice and data collection.

\section{ORCID}

Jeff J. Shi iD http://orcid.org/0000-0002-8529-7100

\section{REFERENCES}

Adams, R. A., \& Thibault, K. M. (2006). Temporal resource partitioning by bats at water holes. Journal of Zoology, 270, 466-472. https://doi. org/10.1111/j.1469-7998.2006.00152.x

Adler, P. B., HilleRisLambers, J., \& Levine, J. M. (2007). A niche for neutrality. Ecology Letters, 10, 95-104. https://doi.org/10.1111/j.14610248.2006.00996.x

Aguirre, L. F., Herrel, A., van Damme, R., \& Matthysen, E. (2002). Ecomorphological analysis of trophic niche partitioning in a tropical savannah bat community. Proceedings of the Royal Society B: Biological Sciences, 269, 1271-1278. https://doi.org/10.1098/rspb.2002.2011

Anacker, B. L., \& Strauss, S. Y. (2014). The geography and ecology of plant speciation: Range overlap and niche divergence in sister species. Proceedings of the Royal Society B: Biological Sciences, 281, 20132980. https://doi.org/10.1098/rspb.2013.2980

Barraclough, T. G., \& Vogler, A. P. (2000). Detecting the geographical pattern of speciation from species-level phylogenies. The American Naturalist, 155, 419-434.

Bengtsson, J. (1989). Interspecific competition increases local extinction rate in a metapopulation system. Nature, 340, 713-715. https://doi. org/10.1038/340713a0
Brown, W. L. Jr., \& Wilson, E. O. (1956). Character displacement. Systematic Zoology, 5, 49-64. https://doi.org/10.2307/2411924

Cardillo, M., \& Warren, D. L. (2016). Analysing patterns of spatial and niche overlap among species at multiple resolutions. Global Ecology and Biogeography, 25, 951-963. https://doi.org/10.1111/geb.12455

Cavender-Bares, J., Kozak, K. H., Fine, P. V. A., \& Kembel, S. W. (2009). The merging of community ecology and phylogenetic biology. Ecology Letters, 12, 693-715. https://doi.org/10.1111/j.1461-0248.2009. 01314.x

Chesson, P. L. (1986). Environmental variation and the coexistence of species. In J. Diamond \& T. J. Case (Eds), Community ecology (pp. 240-256). New York, NY: Harper and Row.

Chesson, P. (2000). Mechanisms of maintenance of species diversity. Annual Review of Ecology and Systematics, 31, 343-366. https://doi. org/10.1146/annurev.ecolsys.31.1.343

Colwell, R. K., \& Lees, D. C. (2000). The mid-domain effect: Geometric constraints on the geography of species richness. Trends in Ecology and Evolution, 15, 70-76. https://doi.org/10.1016/S0169-5347(99) 01767-X

Connell, J. H. (1972). Interactions on marine rocky intertidal shores. Annual Review of Ecology and Systematics, 3, 169-192. https://doi. org/10.1146/annurev.es.03.110172.001125

Corcoran, A. J., \& Conner, W. E. (2014). Bats jamming bats: Food competition through sonar interference. Science, 346, 745-747. https://doi. org/10.1126/science.1259512

Curtis, A. A., \& Simmons, N. B. (2017). Unique turbinal morphology in horseshoe bats (Chiroptera: Rhinolophidae). The Anatomical Record, 300, 309-325. https://doi.org/10.1002/ar.23516

Dumont, E. R. (2004). Patterns of diversity in cranial shape among plantvisiting bats. Acta Chiropterologica, 6, 59-74. https://doi.org/10. 3161/001.006.0105

Dumont, E. R., Dávalos, L. M., Goldberg, A., Santana, S. E., Rex, K., \& Voigt, C. C. (2012). Morphological innovation, diversification and invasion of a new adaptive zone. Proceedings of the Royal Society B: Biological Sciences, 279, 1797-1805. https://doi.org/10.1098/rspb. 2011.2005

Dunson, W., \& Travis, J. (1991). The role of abiotic factors in community organization. The American Naturalist, 138, 1067-1091. https://doi. org/10.1086/285270

Estrada-Villegas, S., McGill, B. J., \& Kalko, E. K. V. (2012). Climate, habitat $\&$ species interactions at different scales determine the structure of a Neotropical bat community. Ecology, 93, 1183-1193. https://doi.org/ 10.1890/11-0275.1

Fenton, M. B., \& Thomas, D. W. (1980). Dry-season overlap in activity patterns, habitat use \& prey selection by sympatric African insectivorous bats. Biotropica, 12, 81-90. https://doi.org/10.2307/ 2387723

Findley, J. S., \& Black, H. A. L. (1983). Morphological and dietary structuring of a Zambian insectivorous bat community. Ecology, 64, 625-630. https://doi.org/10.2307/1937180

Fitzpatrick, B. M., \& Turelli, M. (2006). The geography of mammalian speciation: Mixed signals from phylogenies and range maps. Evolution, 60, 601-615. https://doi.org/10.1111/j.0014-3820.2006.tb01140.x

Fleming, T. H. (1986). Opportunism versus specialization: The evolution of feeding strategies in frugivorous bats. In A. Estrada \& T. H. Fleming (Eds), Frugivores and seed dispersal (pp. 105-118). Dordrecht, Netherlands: Dr W. Junk Publishers. https://doi.org/10.1007/97894-009-4812-9

Graham, C. H., \& Fine, P. V. A. (2008). Phylogenetic beta diversity: Linking ecological and evolutionary processes across space in time. Ecology Letters, 11,1265-1277.https://doi.org/10.1111/j.1461-0248.2008.01256.x

Grant, P. R., \& Grant, B. R. (1997). Genetics and the origin of bird species. Proceedings of the National Academy of Sciences of the United States of America, 94, 7768-7775. https://doi.org/10.1073/pnas.94. 15.7768 
Grossenbacher, D., Briscoe Runquist, R., Goldberg, E. E., \& Brandvain, Y. (2015). Geographic range size is predicted by plant mating system. Ecology Letters, 18, 706-713. https://doi.org/10.1111/ele.12449

Hadfield, J. D. (2010). MCMC methods for multi-response generalized linear mixed models: The MCMCgImm R package. Journal of Statistical Software, 33, 1-22.

Hadfield, J. D., \& Nakagawa, S. (2010). General quantitative genetic methods for comparative biology: Phylogenies, taxonomies and multitrait models for continuous and categorical characters. Journal of Evolutionary Biology, 23, 494-508. https://doi.org/10.1111/j.1420-9101. 2009.01915.x

Heithaus, E. R., Fleming, T. H., \& Opler, P. A. (1975). Foraging patterns and resource utilization in seven species of bats in a seasonal tropical forest. Ecology, 56, 841-854. https://doi.org/10.2307/1936295

Henry, M., Barrière, P., Gautier-Hion, A., \& Colyn, M. (2004). Species composition, abundance and vertical stratification of a bat community (Megachiroptera: Pteropodidae) in a West African rain forest. Journal of Tropical Ecology, 20, 21-29. https://doi.org/10.1017/S02664674 04006145

IUCN. (2016) The IUCN red list of threatened species. Version 2016-2.

Jones, K. E., Bininda-Emonds, O. R. P., \& Gittleman, J. L. (2005). Bats, clocks \& rocks: Diversification patterns in Chiroptera. Evolution, 59, 2243-2255. https://doi.org/10.1111/j.0014-3820.2005.tb00932.x

Jønsson, K. A., Tøttrup, A. P., Borregaard, M. K., Keith, S. A., Rahbek, C., \& Thorup, K. (2016). Tracking animal dispersal: From individual movement to community assembly and global range dynamics. Trends in Ecology and Evolution, 31, 204-214. https://doi.org/10.1016/j.tree. 2016.01.003

Kingston, T., Jones, G., Zubaid, A., \& Kunz, T. H. (2000). Resource partitioning in rhinolophoid bats revisited. Oecologia, 124, 332-342. https://doi.org/10.1007/PL00008866

Kingston, T., \& Rossiter, S. J. (2004). Harmonic-hopping in Wallacea's bats. Nature, 429, 654-657. https://doi.org/10.1038/nature02487

Kneitel, J. M., \& Chase, J. M. (2004). Trade-offs in community ecology: Linking spatial scales and species coexistence. Ecology Letters, 7, 6980. https://doi.org/10.1046/j.1461-0248.2003.00551.x

Kronfeld-Schor, N., \& Dayan, T. (2003). Partitioning of time as an ecological resource. Annual Review of Ecology, Evolution \& Systematics, 34, 153-181. https://doi.org/10.1146/annurev.ecolsys.34.011802.132 435

Leibold, M. A., \& McPeek, M. A. (2006). Coexistence of the niche and neutral perspectives in community ecology. Ecology, 87, 1399-1410. https://doi.org/10.1890/0012-9658(2006)87[1399:COTNAN]2.0. $\mathrm{CO} ; 2$

Lessard, J. P., Belmaker, J., Myers, J. A., Chase, J. M., \& Rahbek, C. (2012). Inferring local ecological processes amid species pool influences. Trends in Ecology and Evolution, 27, 600-607. https://doi.org/ 10.1016/j.tree.2012.07.006

Louthan, A. M., Doak, D. F., \& Angert, A. L. (2015). Where and when do species interactions set range limits? Trends in Ecology and Evolution, 30, 780-792. https://doi.org/10.1016/j.tree.2015.09.011

McCain, C. M. (2007a). Area and mammalian elevational diversity. Ecology, 88, 76-86. https://doi.org/10.1890/0012-9658(2007)88[76: AAMED]2.0.CO;2

McCain, C. M. (2007b). Could temperature and water availability drive elevational species richness patterns? A global case study for bats. Global Ecology and Biogeography, 16, 1-13. https://doi.org/10.1111/j. 1466-8238.2006.00263.x

Mcintire, E. J. B., \& Fajardo, A. (2014). Facilitation as a ubiquitous driver of biodiversity. New Phytologist, 201, 403-416. https://doi.org/10. 1111/nph.12478

McPeek, M. A., \& Brown, J. M. (2000). Building a regional species pool: Diversification of the Enallagma damselflies in eastern North America. Ecology, 81, 904-920. https://doi.org/10.1890/0012-9658(2000)081 [0904:BARSPD]2.0.CO;2
Moreno, C., Arita, H., \& Solis, L. (2006). Morphological assembly mechanisms in Neotropical bat assemblages and ensembles within a landscape. Oecologia, 149, 133-140. https://doi.org/10.1007/ s00442-006-0417-0

Nogueira, M. R., Peracchi, A. L., \& Monteiro, L. R. (2009). Morphological correlates of bite force and diet in the skull and mandible of phyllostomid bats. Functional Ecology, 23, 715-723. https://doi.org/10. 1111/j.1365-2435.2009.01549.x

Norberg, U. M., \& Rayner, J. M. V. (1987). Ecological morphology and flight in bats (Mammalia; Chiroptera): Wing adaptations, flight performance, foraging strategy and echolocation. Philosophical Transactions of the Royal Society B: Biological Sciences, 316, 335-427. https://doi. org/10.1098/rstb.1987.0030

Nowak, M. D. (1994). Walker's bats of the world. Baltimore, MD: Johns Hopkins University Press.

Olson, D. M., Dinerstein, E., Wikramanayake, E. D., Burgess, N. D., Powell, G. V. N., Underwood, E. C., ... Kassem, K. R. (2001). Terrestrial ecoregions of the world: A new map of life on Earth. BioScience, 51, 933-938. https://doi.org/10.1641/0006-3568(2001)051[0933:TEOT WA]2.0.CO;2

Phillimore, A. B., Orme, C. D. L., Thomas, G. H., Blackburn, T. M., Bennett, P. M., Gaston, K. J., \& Owens, I. P. F. (2008). Sympatric speciation in birds is rare: Insights from range data and simulations. The American Naturalist, 171, 646-657. https://doi.org/10.1086/587074

Pigot, A. L., \& Tobias, J. A. (2013). Species interactions constrain geographic range expansion over evolutionary time. Ecology Letters, 16, 330-338. https://doi.org/10.1111/ele.12043

Pigot, A. L., \& Tobias, J. A. (2014). Dispersal and the transition to sympatry in vertebrates. Proceedings of the Royal Society B: Biological Sciences, 282, 20141929. https://doi.org/10.1098/rspb.2014.1929

Rabosky, D. L., Cowan, M. A., Talaba, A. L., \& Lovette, I. J. (2011). Species interactions mediate phylogenetic community structure in a hyperdiverse lizard assemblage from arid Australia. The American Naturalist, 178, 579-595. https://doi.org/10.1086/662162

Ricklefs, R. E. (2007). History and diversity: Explorations at the intersection of ecology and evolution. The American Naturalist, 170(Suppl), S56-S70. https://doi.org/10.1086/519402

Santana, S. E., \& Cheung, E. (2016). Go big or go fish: Morphological specializations in carnivorous bats. Proceedings of the Royal Society B: Biological Sciences, 283, 20160615. https://doi.org/10.1098/rspb. 2016.0615

Santana, S. E., Dumont, E. R., \& Davis, J. L. (2010). Mechanics of bite force production and its relationship to diet in bats. Functional Ecology, 24, 776-784. https://doi.org/10.1111/j.1365-2435.2010.01703. $\mathrm{x}$

Santana, S. E., \& Lofgren, S. E. (2013). Does nasal echolocation influence the modularity of the mammal skull? Journal of Evolutionary Biology, 26, 2520-2526. https://doi.org/10.1111/jeb.12235

Saunders, M. B., \& Barclay, R. M. R. (1992). Ecomorphology of insectivorous bats: A test of predictions using two morphologically similar species. Ecology, 73, 1335-1345. https://doi.org/10.2307/1940680

Schoeman, M. C., \& Jacobs, D. S. (2003). Support for the allotonic frequency hypothesis in an insectivorous bat community. Oecologia, 134, 154-162. https://doi.org/10.1007/s00442-002-1107-1

Schoeman, M. C., \& Jacobs, D. S. (2011). The relative influence of competition and prey defences on the trophic structure of animalivorous bat ensembles. Oecologia, 1, 493-506. https://doi.org/10.1007/ s00442-010-1854-3

Sexton, J. P., McIntyre, P. J., Angert, A. L., \& Rice, K. J. (2009). Evolution and ecology of species range limits. Annual Review of Ecology, Evolution, and Systematics, 40, 415-436. https://doi.org/10.1146/annure v.ecolsys.110308.120317

Shi, J. J., \& Rabosky, D. L. (2015). Speciation dynamics during the global radiation of extant bats. Evolution, 69, 1528-1545. https://doi.org/ 10.1111/evo.12681 
Siemers, B. M., \& Schnitzler, H.-U. (2004). Echolocation signals reflect niche differentiation in five sympatric congeneric bat species. Nature, 429, 657-661. https://doi.org/10.1038/nature02547

Silvestro, D., Antonelli, A., Salamin, N., \& Quental, T. B. (2015). The role of clade competition in the diversification of North American canids. Proceedings of the National Academy of Sciences of the United States of America, 112, 8684-8689. https://doi.org/10.1073/pnas.15028 03112

Simmons, N. B. (2005). Order Chiroptera. In D. E. Wilson, \& D. M. Reeder (Eds.), Mammal species of the world: A taxonomic and geographic reference (3rd ed., pp. 312-529). Baltimore, MD: Johns Hopkins University Press.

Simmons, N. B., \& Conway, T. M. (2003). Evolution of ecological diversity in bats. In T. H. Kunz, \& M. B. Fenton (Eds.), Bat ecology (pp. 493535). Chicago, IL: University of Chicago Press.

Spiesman, B. J., \& Inouye, B. D. (2014). The consequences of multiple indirect pathways of interaction for species coexistence. Theoretical Ecology, 8, 225-232.

Stuart, Y. E., \& Losos, J. B. (2013). Ecological character displacement: Glass half full or half empty? Trends in Ecology and Evolution, 28, 402-408. https://doi.org/10.1016/j.tree.2013.02.014

Swift, S. M., \& Racey, P. A. (1983). Resource partitioning in two species of vespertilionid bats (Chiroptera) occupying the same roost. Journal of Zoology, 200, 249-259.

Taylor, E. B., Boughman, J. W., Groenenboom, M., Sniatynski, M., Schluter, D., \& Gow, J. L. (2006). Speciation in reverse: Morphological and genetic evidence of the collapse of a three-spined stickleback (Gasterosteus aculeatus) species pair. Molecular Ecology, 15, 343-355.

Terribile, L. C., Diniz-Filho, J. A. F., Rodríguez, M. Á., \& Rangel, T. F. L. V. B. (2009). Richness patterns, species distributions and the principle of extreme deconstruction. Global Ecology and Biogeography, 18, 123136. https://doi.org/10.1111/j.1466-8238.2008.00440.x

Tobias, J. A., Cornwallis, C. K., Derryberry, E. P., Claramunt, S., Brumfield, R. T., \& Seddon, N. (2014). Species coexistence and the dynamics of phenotypic evolution in adaptive radiation. Nature, 506, 359-363. https://doi.org/10.1038/nature12874

Villalobos, F., \& Arita, H. T. (2010). The diversity field of New World leaf-nosed bats (Phyllostomidae). Global Ecology and Biogeography, 19, 200-211. https://doi.org/10.1111/j.1466-8238.2009.00503.x

Voss, R. S., Fleck, D. W., Strauss, R. E., Velazco, P. M., \& Simmons, N. B. (2016). Roosting ecology of Amazonian bats: Evidence for guild structure in hyperdiverse mammalian communities. American Museum Novitates, 3870, 1-43. https://doi.org/10.1206/3870.1

Warren, D. L., Cardillo, M., Rosauer, D. F., \& Bolnick, D. I. (2014). Mistaking geography for biology: Inferring processes from species distributions. Trends in Ecology and Evolution, 29, 572-580. https://d oi.org/10.1016/j.tree.2014.08.003

Webb, C. O. (2000). Exploring the phylogenetic structure of ecological communities: An example for rain forest trees. The American Naturalist, 156, 145-155. https://doi.org/10.1086/303378

Weir, J. T., \& Price, T. D. (2011). Limits to speciation inferred from times to secondary sympatry and ages of hybridizing species along a latitudinal gradient. The American Naturalist, 177, 462-469. https://doi. org/10.1086/658910

\section{BIOSKETCH}

Jeff J. Shi performed this research as part of his Ph.D. in Ecology and Evolutionary Biology at the University of Michigan. His dissertation evaluates how extant patterns of diversity reflect dynamic histories of diversification and species interactions; he focuses on extant bats as a model study system.

Author contributions: J.J.S. and D.L.R. designed this study and its methods and analyses, and led the writing of the manuscript. J.J.S., E.P.W., N.T.K. and E.R.D. contributed to data collection. E.P.W. and E.R.D. reviewed, added to and commented on the manuscript throughout the writing process.

\section{SUPPORTING INFORMATION}

Additional supporting information may be found online in the Supporting Information section at the end of the article.

How to cite this article: Shi JJ, Westeen EP, Katlein NT, Dumont ER, Rabosky DL. Ecomorphological and phylogenetic controls on sympatry across extant bats. J Biogeogr. 2018;45:1560-1570. https://doi.org/10.1111/jbi.13353 